\title{
ON THE THEORY OF $\Pi_{3}^{1}$ SETS OF REALS
}

\author{
BY A. S. KECHRIS ${ }^{1}$ AND D. A. MARTIN
}

Communicated by Solomon Feferman, June 2, 1977

1. An ordinal basis theorem. Assuming that $\forall x \in \omega^{\omega}$ ( $x^{\#}$ exists), let $u_{\alpha}$ be the $\alpha$ th uniform indiscernible (see [3] or [2]). A canonical coding system for ordinals $\left\langle u_{\omega}\right.$ can be defined by letting $w_{0}=\left\{w \in \omega^{\omega}: w=\left\langle n, x^{\#}\right\rangle\right.$, for some $\left.n \in \omega, x \in \omega^{\omega}\right\}$ and for $w=\left\langle n, x^{\#}\right\rangle \in W 0_{\omega},|w|=\tau_{n}^{L}[x]\left(u_{1}, \ldots, u_{k_{n}}\right)$, where $\tau_{n}$ is the $n$th term in a recursive enumeration of all terms in the language of $Z F+V=L[\dot{x}], \dot{x}$ a constant, taking always ordinal values. Call a relation $P(\xi, x)$, where $\xi$ varies over $u_{\omega}$ and $x$ over $\omega^{\omega}, \Pi_{k}^{1}$ if $P^{*}(w, x) \Leftrightarrow w \in W 0_{\omega} \wedge$ $P(|w|, x)$ is $\Pi_{k}^{1}$. An ordinal $\xi<u_{\omega}$ is called $\Delta_{k}^{1}$ if it has a $\Delta_{k}^{1}$ notation i.e. $\exists w$ $\in W 0_{\omega}\left(w \in \Delta_{k}^{1} \wedge|w|=\xi\right)$.

THEOREM $1\left(Z F+D C+\right.$ DeterminaCy $\left.\left(\Delta_{2}^{1}\right)\right)$. Every nonempty $\Pi_{3}^{1}$ subset of $u_{\omega}$ contains a $\Delta_{3}^{1}$ ordinal.

Corollary $2\left(Z F+D C+\right.$ Determinacy $\left.\left(\Delta_{2}^{1}\right)\right)$. $\Pi_{3}^{1}$ is closed under quantification over ordinals $<u_{\omega}$ i.e. if $P(\xi, x)$ is $\Pi_{3}^{1}$ so are $\exists \xi P(\xi, x), \forall \xi P(\xi, x)$.

Corollary $3(Z F+D C+A D)$. The class of $\Pi_{3}^{1}$ sets of reals is closed under $<\delta_{3}^{1}$ intersections and unions.

Martin [3] has proved the corresponding result for $\Delta_{\mathbf{3}}^{\mathbf{1}}$.

2. A Kleene theory for $\Pi_{3}^{1}$. Kleene has characterized the $\Pi_{1}^{1}$ relations as those which are inductive (see [7]) on the structure $\left\langle\omega,\langle\rangle=Q_{1}\right.$. Let $j_{m}: u_{\omega}$ $\rightarrow u_{\omega}, m \geqslant 1$, be defined by letting

$$
j_{m}\left(u_{i}\right)= \begin{cases}u_{i}, & \text { if } i<m, \\ u_{i+1}, & \text { if } i \geqslant m,\end{cases}
$$

and then

$$
j_{m}\left(\tau_{n}^{L[x]}\left(u_{1}, \cdots u_{k_{n}}\right)\right)=\tau_{n}^{L[x]}\left(j_{m}\left(u_{1}\right) \cdots j_{m}\left(u_{k_{n}}\right)\right) .
$$

Let $R$ be the relation on $u_{\omega}$ coding these embeddings, i.e.

$$
R=\left\{(m, \alpha, \beta): m \in \omega \wedge \alpha, \beta<u_{\omega} \wedge j_{m}(\alpha)=\beta\right\}
$$

Put $Q_{3}=\left\langle u_{\omega},\langle, R\rangle\right.$.

AMS (MOS) subject classifications (1970). Primary 04A15, 02K30, 28A05, 54H05; Secondary 02F35, $02 \mathrm{K25}, 02 \mathrm{K35}, 04 \mathrm{A30}$.

${ }^{1}$ Research partially supported by NSF Grant MCS 76-17254. 
THEOREM 4. $\left(Z F+D C+\right.$ Determinacy $\left.\left(\Delta_{2}^{1}\right)\right)$. A set of reals is $\Pi_{3}^{1}$ iff it is absolutely inductive on the structure $2_{3}$.

In the second part of the above characterization a relation on reals is viewed as a second order relation on $u_{\omega}$ and absolutely inductive means that only parameters from $\omega$ are allowed in the definitions (see [7]).

It should be mentioned here that $Q_{3}$ is up to absolute hyperelementary equivalence the same as $\left\langle u_{\omega},<, T^{2}\right\rangle$, where $T^{2}$ is the tree (on $\omega \times u_{\omega}$ ) coming from the Martin and Solovay [4] analysis of $\Pi_{2}^{1}$ sets (see [3] for the definition of $\left.T^{2}\right)$.

One also obtains the analog for $\Pi_{3}^{1}$ of the Souslin-Kleene representation of $\Pi_{1}^{1}$ sets in terms of well-founded trees.

Theorem $5\left(Z F+D C+\right.$ Determinacy $\left.\left(\Delta_{2}^{1}\right)\right)$. A set of reals $P$ is $\Pi_{3}^{1}$ iff there is a tree $T$ on $\omega \times u_{\omega}$ which is recursive in the structure $Q_{3}$ and $P(x) \Leftrightarrow$ $T(x)$ is well founded.

For the notation see [2]. The fact that every $\Pi_{3}^{1}$ set can be so represented is a well-known result of Martin and Solovay [4], the converse being new here.

Let $2 \overline{3}=\left\langle u_{\omega},<,\left\{u_{n}\right\}_{n<\omega}\right\rangle$. Then we also have the context of full $A D$, in which case $u_{n}=\aleph_{n}, \forall n \leqslant \omega$.

Theorem $6(Z F+D C+A D)$. A set of reals is $\Pi_{3}^{1}$ iff it is $\Pi_{1}^{1}$ on the structure $2 \overline{3}^{-}$.

3. Explaining the $Q$-theory. The results in $\$ 2$ provide a nice explanation for the $Q$-theory (see [5], [1]) at level 3, which accounts for the structural differences between $\Pi_{3}^{1}$ and $\Pi_{1}^{1}$ sets. For example, a real is $\Delta_{3}^{1}$ iff it is absolutely hyperelementary on $Q_{3}$ while it is in $Q_{3}$ iff it is hyperelementary (i.e. parameters $<u_{\omega}$ are allowed) on $Q_{3}$. Also if $y_{0}$ is the first nontrivial $\Pi_{3}^{1}$ singleton then $y_{0}$ is hyperelementary-in- $Q_{3}$ equivalent to the complete inductive-in- $Q_{3}$ subset of $u_{\omega}$.

4. Higher level analogs of $L$. Assuming Projective Determinacy (PD), let $T^{3}$ be the tree (on $\omega \times \delta_{3}^{1}$ ) associated with an arbitrary $\Pi_{3}^{1}$-scale on a complete $\Pi_{3}^{1}$ set (see [6] and [2]). Let also $C_{4}$ be the largest countable $\Sigma_{4}^{1}$ set. The next result proves a conjecture of Moschovakis and shows that $L\left[T^{3}\right]$ is a correct higher level analog of $L$ for level 4 .

TheOREM $7\left(Z F+D C+\right.$ Determinacy $\left(L\left[\omega^{\omega}\right] \cap\right.$ power $\left.\left.\left(\omega^{\omega}\right)\right)\right)$. For any $T^{3}$ as above, $L\left[T^{3}\right] \cap \omega^{\omega}=C_{4}$. In particular $L\left[T^{3}\right] \cap \omega^{\omega}$ is independent of the tree $T^{3}$.

Open problem. Is $L\left[T^{3}\right]$ independent of $T^{3}$ ?

Further applications of the methods developed here to the theory of $\Pi_{3}^{1}$ sets as well as details and proofs of the results announced here will appear elsewhere. 


\section{REFERENCES}

1. A. S. Kechris, The theory of countable analytical sets, Trans. Amer. Math. Soc. 202 (1975), 259-297.

2. On projective ordinals, J. Symbolic Logic 39 (1974), 269-282.

3. D. A. Martin, Projective sets and cardinal numbers; some questions related to the continuum problem, J. Symbolic Logic (to appear).

4. D. A. Martin and R. M. Solovay, A basis theorem for $\Sigma_{3}^{1}$ sets of reals, Ann. of Math. 89 (1969), 138-160.

5. - Basis theorems for $\Pi_{2 k}^{1}$ sets of reals (to appear).

6. Y. N. Moschovakis, Uniformization in a playful universe, Bull. Amer. Math. Soc. 77 (1970), $731-736$. 1974.

7. - Elementary induction on abstract structures, North-Holland, Amsterdam,

\section{DEPARTMENT OF MATHEMATICS, CALIFORNIA INSTITUTE OF TECHNOLOGY, PASADENA, CALIFORNIA 91125}

DEPARTMENT OF MATHEMATICS, ROCKEFELLER UNIVERSITY, NEW YORK, NEW YORK 10021

Current address (D. A. Martin): Department of Mathematics, University of California, Los Angeles, California 90024 